\title{
Asymmetric anionic polymerization of tris(trimethylsilyl)silyl methacrylate: a highly isotactic helical chiral polymer
}

\author{
Kenji Ishitake $^{1}$, Kotaro Satoh ${ }^{1}$, Masami Kamigaito ${ }^{1}$ and Yoshio Okamoto ${ }^{2,3}$ \\ Polymer Journal (2013) 45, 676-680; doi:10.1038/pj.2012.178; published online 17 October 2012
}

Keywords: anionic polymerization; asymmetric polymerization; chiral polymer; helical polymer; isotactic polymer; methacrylate; supersilyl group

\section{INTRODUCTION}

Triarylmethyl methacrylate and related bulky methacrylates, such as triphenylmethyl (TrMA) methacrylate and 1-phenyldibezosuberyl methacrylate (PDBSMA), are known to yield highly isotactic and optically active polymers having a stable one-handed helical conformation via asymmetric anionic polymerization with a chiral initiating system. ${ }^{1-8}$ The bulky triarylmethyl substituent dictates the helical conformation by forcing the chain end to propagate in an isospecific manner, and the chiral ligand or initiator determines the helix sense. These polymers show a high chiral recognition ability for racemic compounds and are utilized as chiral stationary phases for high-performance liquid chromatography. ${ }^{9-12}$ However, all of these bulky substituents that are effective in helix-sense-selective and highly isotactic polymerizations are based on the triarylmethyl substituent or related skeletons; ${ }^{13-24}$ no other effective groups have been identified previously.

The tris(trimethylsilyl)silyl (TTMSS) group, also called the hypersilyl, sisyl, or supersilyl group, ${ }^{25}$ is an extremely bulky silyl group and has recently been used to induce diastereoselectivity in various reactions, such as $(2+2)$ cyclizations, Mukaiyama aldol reactions and cascade reactions of its enol ether by Yamamoto et al. ${ }^{26-29}$ These bulky silyl ethers are stable under ionic conditions and can undergo various stereoselective organic reactions due to their highly bulky nature. Recently, we succeeded in synthesizing the novel highly bulky silyl methacrylate tris(trimethylsilyl)silyl methacrylate (TTMSSMA) from the sodium salt of methacrylic acid and supersilyl chloride. We also achieved highly isotactic radical polymerization $(\mathrm{mm}>90 \%)$ with this new methacrylate, for which the isotacticity is higher than that for TrMA and is slightly lower than that for PDBSMA. ${ }^{30}$ We also achieved the facile transformation of the resulting polymers into isotactic poly(methacrylic acid) (PMAA) by removal of the silyl group. ${ }^{31}$ These results suggest that the TTMSS group in the methacrylate is sufficiently bulky to dictate the isotactic radical propagation, as is done by the triaryl group. The anionic polymerization of this novel bulky silyl methacrylate has not been studied previously. In contrast to the bulky silyl monomer, trimethylsilyl methacrylate, a protected methacrylic acid with a small silyl group, can be polymerized under anionic conditions ${ }^{32-36}$ to give stereoregular polymers that depend on the anionic initiating system. $^{37,38}$

This study is thus focused on the asymmetric anionic polymerization of TTMSSMA using an anionic initiator in the presence of a chiral ligand to develop a novel bulky methacrylate that will produce optically active polymers with a one-handed helical structure (Scheme 1). The initiating system consisting of 9-fluorenyllithium (Fl-Li) and ( -$)$-sparteine $((-)-\mathrm{Sp})$ induced the asymmetric anionic polymerization of TTMSSMA quantitatively, yielding insoluble but highly isotactic $(m m>99 \%)$ optically active polymers, as confirmed by diffuse reflectance circular dichroism (DRCD) spectroscopy. ${ }^{39,40}$

\section{EXPERIMENTAL PROCEDURE}

\section{Materials}

Fluorene (TCI; Tokyo Chemical Industry Co., Ltd., Tokyo, Japan, >95\%) was purified by recrystallization from hexane twice. $(S, S)-(+)-2,3$-Dimethoxy1,4-bis(dimethylamino)butane ((+)-DDB) (TCI, >97\%), $(R, R)-(-)-2,3-$ dimethoxy-1,4-bis(dimethylamino)butane $((-)-\mathrm{DDB}) \quad(\mathrm{TCI},>90 \%)$ and 1,2,3,4-tetrahydronaphthalene (WAKO; Wako Pure Chemical Industries, Ltd., Osaka, Japan, 97\%) were distilled from calcium hydride under reduced pressure before use. ( -$)$-Sparteine $((-)-S p)$ was obtained by washing $(-)$ sparteine sulfate pentahydrate (WAKO, 98\%) with $\mathrm{NaOH}$ a.q. and distilled from calcium hydride under reduced pressure before use. Toluene (Kanto; Kanto Chemical Co. Inc., Tokyo, Japan, $>99.5 \% ; \mathrm{H}_{2} \mathrm{O}<10$ p.p.m.) and tetrahydrofuran (THF; Kanto, $>99.5 \% ; \mathrm{H}_{2} \mathrm{O}<10$ p.p.m.) were dried and

${ }^{1}$ Department of Applied Chemistry, Graduate School of Engineering, Nagoya University, Nagoya, Japan; ${ }^{2}$ Nagoya University, Nagoya, Japan and ${ }^{3}$ College of Material Science and Chemical Engineering, Harbin Engineering University, Harbin, China

Correspondence: Professor M Kamigaito, Department of Applied Chemistry, Nagoya University, Graduate School of Engineering, Furo-cho, Chikusa-ku, Nagoya 464-8603, Japan.

E-mail: kamigait@apchem.nagoya-u.ac.jp

Received 7 May 2012; revised 19 July 2012; accepted 7 September 2012; published online 17 October 2012 


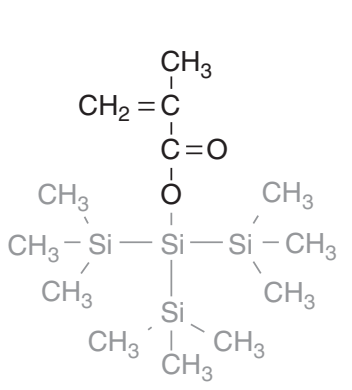

Tris(trimethylsilyl)silyl Methacrylate

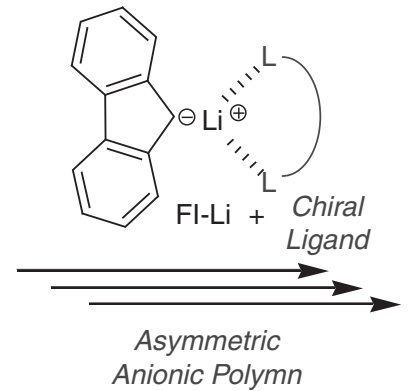

Anionic Polymn

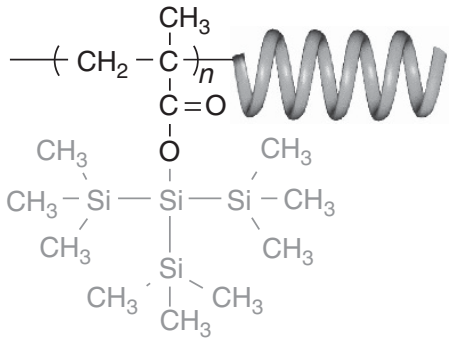

Helical Chiral Polymer

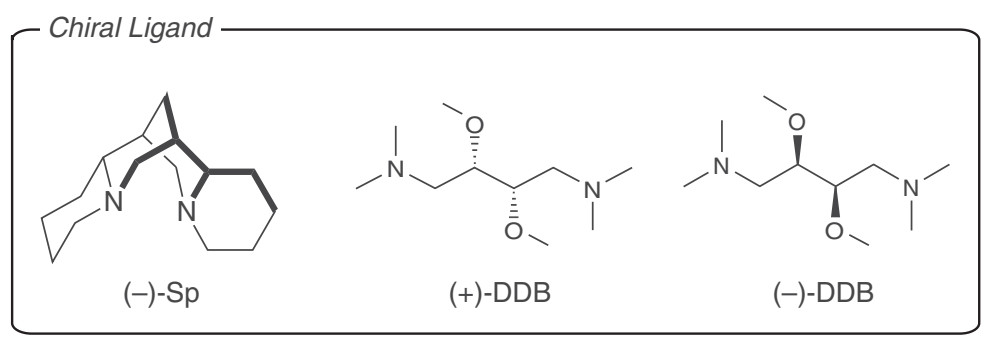

Scheme 1 Asymmetric anionic polymerization of supersilyl methacrylate. A full color version of this figure is available at Polymer Journal online.

deoxygenized by passage through columns of Glass Contour Solvent Systems (Pure Process Technology, Nashua, NH, USA) before use. Trimethylsilyl diazomethane (Sigma-Aldrich, St Louis, MO, USA, $2.0 \mathrm{M}$ in $\mathrm{Et}_{2} \mathrm{O}$ ), tetrabutylammonium fluoride (Sigma-Aldrich, $1.0 \mathrm{M}$ in THF) and $n \mathrm{BuLi}$ (Kanto, $1.57 \mathrm{M}$ in $n$-hexane) were used as received. TTMSSMA was synthesized according to the literature. ${ }^{31}$

\section{General procedure for asymmetric anionic polymerization}

Polymerization was carried out by the syringe technique under dry argon in sealed glass tubes. A typical example for polymerization of TTMSSMA with Fl$\mathrm{Li}$ in toluene is given below. Fl-Li was prepared by adding $n \mathrm{BuLi}(1.00 \mathrm{ml}$ of $1.57 \mathrm{M}$ solution in $n$-hexane) to a solution of fluorene $(0.261 \mathrm{~g}, 1.57 \mathrm{mmol})$ in toluene $(14.0 \mathrm{ml})$ at room temperature. The Fl- $\mathrm{Li}$ solution was mixed with ( -)-Sp ( $0.43 \mathrm{ml}, 1.87 \mathrm{mmol})$ and then left for $10 \mathrm{~min}$ at room temperature to prepare the chiral initiator solution. In another glass tube were placed toluene $(0.94 \mathrm{ml})$ and toluene solution of TTMSSMA $(0.86 \mathrm{ml}$ of $1.16 \mathrm{M}, 7.00 \mathrm{mmol})$ containing a small amount of 1,2,3,4-tetrahydronaphthalene $(0.05 \mathrm{ml})$ as an internal standard at room temperature to obtain the monomer solution. Then, the monomer solution was cooled at $-78{ }^{\circ} \mathrm{C}$, into which a prescribed amount of an initiator solution $(0.20 \mathrm{ml})$ was added. In predetermined intervals, the polymerization was terminated by the addition of a small amount of degassed methanol $(1.0 \mathrm{ml})$ to the reaction mixtures. Monomer conversion was determined from the concentration of residual monomer measured by ${ }^{1} \mathrm{H}$ nuclear magnetic resonance (NMR) with 1,2,3,4-tetrahydronaphthalene as an internal standard (for example, 96\% conversion in $48 \mathrm{~h}$ ). The quenched reaction solutions were washed with $n$-hexane and then dried to give poly(TTMSSMA).

\section{Transformation of poly(TTMSSMA) into PMAA and poly(methyl methacrylate)}

The obtained poly(TTMSSMA)s were converted into PMAA and poly(methyl methacrylate) (PMMA). The silyl groups in poly(TTMSSMA) were deprotected by tetrabutylammonium fluoride. A typical example for poly (TTMSSMA) by deprotection of the silyl group followed by methylation with trimethylsilyl diazomethane is as follows: a portion of the poly(TTTMSSMA) (50 mg) obtained with Fl-Li/( -)-Sp was freeze dried with benzene and a THF solution of tetrabutylammonium fluoride $(1.0 \mathrm{M}, 5.0 \mathrm{ml})$ was added dropwise at $0{ }^{\circ} \mathrm{C}$ over a period of $10 \mathrm{~min}$ under stirring. The solution was stirred at $40{ }^{\circ} \mathrm{C}$ for an additional $24 \mathrm{~h}$. The deprotection was quenched by adding methanol $(5.0 \mathrm{ml})$. After concentrating it by evaporation, the product was dissolved in
$20 \mathrm{ml}$ of a toluene/ $\mathrm{CH}_{3} \mathrm{OH}$ mixture (4/1 volume) and then an $\mathrm{Et}_{2} \mathrm{O}$ solution of trimethylsilyl diazomethane $(2.0 \mathrm{M}, 5.0 \mathrm{ml})$ was added. After $12 \mathrm{~h}$, the methylation was quenched by adding a small amount of acetic acid. The mixture was washed with distilled water and evaporated to dryness under reduced pressure, and then vacuum-dried to give PMMA ( $12 \mathrm{mg}, M_{\mathrm{n}}=37500$, $\left.M_{\mathrm{w}} / M_{\mathrm{n}}=2.20\right)$ containing a small amount of the residual silyl fluoride.

Polymer samples for NMR analysis were fractionated by preparative sizeexclusion chromatography (column: Shodex K-2002; SHOWA DENKO K.K., Tokyo, Japan) to be free from low-molecular weight compounds without the loss of MMA oligomers if present.

\section{Chiral absorption}

A finely ground polymer $(15 \mathrm{mg})$ was put in a $2-\mathrm{ml}$ screw-capped glass vial. A solution of a racemic compound in methanol $(200 \mu$ l, concentration $=0.25$ $\mathrm{g} \mathrm{ml}^{-1}$ ) was added to the polymer. The mixture was allowed to stand for $12 \mathrm{~h}$ in a tightly capped container. The e.e. of the analyte in the sample solution was analyzed using the supernatant solution $(10 \mu \mathrm{l})$ by an high-pressure liquid chromatography system equipped with a Jasco UV-970 UV detector, a Jasco (Hachioji, Japan) OR-990 polarimeter, and a Daicel (Osaka, Japan) Chiralcel OD column $(0.46 \mathrm{~cm}$ i.d. $\times 25 \mathrm{~cm})$.

\section{Measurements}

Monomer conversion was determined from the concentration of residual monomer measured by ${ }^{1} \mathrm{H}$ NMR spectroscopy with 1,2,3,4-tetrahydronaphthalene or toluene as an internal standard. ${ }^{1} \mathrm{H}$ NMR spectra for monomer conversion were recorded in $\mathrm{CDCl}_{3}$ at $25^{\circ} \mathrm{C}$ on a Varian (Palo Alto, CA, USA) Mercury 300 spectrometer, operating at $300 \mathrm{MHz} .{ }^{1} \mathrm{H}$ and ${ }^{13} \mathrm{C}$ NMR spectra of the obtained polymers were recorded in $\mathrm{CDCl}_{3}$ at $55^{\circ} \mathrm{C}$ on a JEOL (Akishima, Japan) ECS-400 spectrometer, operating at 400 and $100 \mathrm{MHz}$ for ${ }^{1} \mathrm{H}$ and ${ }^{13} \mathrm{C}$, respectively. The triad tacticity of the polymer was determined by the area of the $\alpha$-methyl protons at $0.8-1.3$ p.p.m. in the ${ }^{1} \mathrm{H}$ NMR spectrum or carbonyl $\mathrm{C}=\mathrm{O}$ carbons at $175-180$ p.p.m. in the ${ }^{13} \mathrm{C}$ NMR spectrum of the side chain. The number-average molecular weight $\left(M_{\mathrm{n}}\right)$ and weight-average molecular weight $\left(M_{\mathrm{w}}\right)$ of the product polymers were determined by sizeexclusion chromatography in $\mathrm{CHCl}_{3}$ at $40{ }^{\circ} \mathrm{C}$ on two polystyrene gel columns (Shodex K-805 (pore size: $20-1000 \AA ̊$; $8.0 \mathrm{~mm}$ i.d. $\times 30 \mathrm{~cm}$ ) $\times 2$; flow rate $1.0 \mathrm{ml} \mathrm{min}^{-1}$ ) connected to Jasco PU-2080 precision pump and a Jasco RI2031 detector. The columns were calibrated against eight standard PMMA samples (Shodex; $M_{\mathrm{p}}=875-1950000 ; M_{\mathrm{w}} / M_{\mathrm{n}}=1.02-1.09$ ). DRCD spectra were measured on a Jasco J-800 spectropolarimeter with a Jasco PCD-466 
Table 1 Anionic polymerization of TTMSSMA ${ }^{a}$

\begin{tabular}{|c|c|c|c|c|c|c|c|c|c|}
\hline Entry & Initiator/additive & Temperature $\left({ }^{\circ} \mathrm{C}\right)$ & {$[\mathrm{M}]_{0} /[\mathrm{I}]_{0}$} & Time (h) & Conversion $(\%)^{\mathrm{b}}$ & $\mathrm{M}_{n}^{\mathrm{c}, \mathrm{d}}$ & $\mathrm{M}_{w} / \mathrm{M}_{n}^{\mathrm{c}, \mathrm{d}}$ & $f(\%)^{e}$ & $\mathrm{~mm} / \mathrm{mr} / \mathrm{rr}$ \\
\hline 1 & $\mathrm{Fl}-\mathrm{Li}$ & -78 & 50 & 27 & 17 & 66700 & 1.87 & 1.5 & $95.8 / 2.7 / 1.5$ \\
\hline $2^{f}$ & $\mathrm{FI}-\mathrm{Li}$ & -78 & 50 & 24 & $>99$ & 11400 & 1.67 & 45 & $90.2 / 7.1 / 2.7$ \\
\hline 3 & $\mathrm{FI}-\mathrm{Li} /(+)-\mathrm{DDB}$ & -78 & 50 & 144 & 4 & - & - & - & - \\
\hline 4 & $\mathrm{Fl}-\mathrm{Li} /(-)-\mathrm{Sp}$ & -78 & 50 & 24 & 96 & 37500 & 2.22 & 13 & $99.4 / 0.4 / 0.2$ \\
\hline 5 & $\mathrm{FI}-\mathrm{Li} /(-)-\mathrm{Sp}$ & -78 & 30 & 24 & $>99$ & 27700 & 2.41 & 3.5 & $99.1 / 0.7 / 0.2$ \\
\hline 6 & $\mathrm{Fl}-\mathrm{Li} /(-)-\mathrm{Sp}$ & -78 & 10 & 24 & $>99$ & 13500 & 2.25 & 8.6 & $99.2 / 0.6 / 0.2$ \\
\hline 7 & $\mathrm{FI}-\mathrm{Li} /(-)-\mathrm{Sp}$ & -40 & 50 & 0.5 & $>99$ & 26300 & 1.84 & 19 & $95.8 / 2.2 / 2.0$ \\
\hline 8 & $\mathrm{FI}-\mathrm{Li} /(+)-\mathrm{DDB}$ & -40 & 50 & 5 & 95 & 8400 & 1.48 & 59 & $61.7 / 23.3 / 15.0$ \\
\hline 9 & $\mathrm{FI}-\mathrm{Li} /(-)-\mathrm{DDB}$ & -40 & 50 & 5 & $>99$ & 6100 & 1.74 & 85 & $61.3 / 23.6 / 15.1$ \\
\hline
\end{tabular}

Abbreviation: TTMSSMA, tris(trimethylsilyl)silyl methacrylate.

apolymerization condition: $[\text { TTMSSMA }]_{0}=0.50 \mathrm{~m}$ in toluene, $[\mathrm{FI}-\mathrm{Li}]_{0} /[$ chiral ligand $]=1.0 / 1.2$.

${ }^{\mathrm{b}} \mathrm{By}{ }^{1} \mathrm{H}$ nuclear magnetic resonance.

'By size-exclusion chromatography (SEC).

${ }^{\mathrm{d}}$ After converted to poly(methyl methacrylate).

e $f$ (initiator efficiency) $=M_{\mathrm{n}}$ (calculated) $/ M_{\mathrm{n}}(\mathrm{SEC})$.

fIn tetrahydrofuran.

apparatus for the poly(TTMSSMA) sample $(15 \mathrm{mg})$ grounded with $130 \mathrm{mg}$ $\mathrm{KCl}$ in pressed quartz plate.

\section{RESULTS AND DISCUSSION}

\section{Anionic polymerization of TTMSSMA}

The anionic polymerization of TTMSSMA in the absence of chiral ligands was first investigated with Fl-Li as an initiator in toluene and THF at $-78^{\circ} \mathrm{C}$ (entries 1 and 2 in Table 1). The monomer was polymerized faster in THF than in toluene (Figure 1a), a result that is consistent with these solvents' effects on the anionic polymerization of $\mathrm{MMA}^{41}$ and $\mathrm{TrMA}^{42}$ in which the propagating anion is more reactive in THF because of the coordination of THF to the countercation, $\mathrm{Li}^{+}$. All of the obtained polymers were insoluble, similar to those obtained in the radical polymerization, ${ }^{31}$ most likely because of the rigid helical structure.

The obtained poly(TTMSSMA)s were then converted into PMMAs to measure the molecular weight and tacticity; this conversion was performed via the deprotection of the silyl groups, followed by methylation. ${ }^{31}$ The molecular weights based on PMMA calibration by size-exclusion chromatography were higher than the calculated values assuming that one molecule of Fl-Li generates one polymer chain. The initiator efficiency obtained in toluene $(1.5 \%)$ was much lower than that in THF (45\%) because of the more pronounced aggregation of the initiator in the nonpolar toluene. As shown in the ${ }^{1} \mathrm{H}$ NMR spectra of the $\alpha$-methyl protons of the obtained PMMAs (Figure 1b), the polymers obtained from the anionic polymerization also possessed a high isotacticity, which depended on the solvent $(\mathrm{mm}=95 \%$ in toluene and $90 \%$ in THF). Similar high isotacticities for anionic polymerizations have also been reported for triarylmethyl methacrylate, ${ }^{15}$ suggesting that helical polymers are formed from TTMSSMA.

\section{Asymmetric anionic polymerization of TTMSSMA}

The asymmetric anionic polymerization of TTMSSMA was then investigated with Fl-Li in the presence of chiral ligands, such as $(-)-\mathrm{Sp}^{1}$ and $(+)-\mathrm{DDB},{ }^{14}$ which are effective in the asymmetric anionic polymerization of bulky triarylmethyl methacrylate. These polymerizations were performed in toluene at $-78^{\circ} \mathrm{C}$ (entries 3 and 4 in Table 1). The Fl-Li/(-)-Sp system induced quantitative polymerization, resulting in insoluble polymers, whereas no polymerization occurred with the $\mathrm{Fl}-\mathrm{Li} /(+)$-DDB system at $-78^{\circ} \mathrm{C}$.
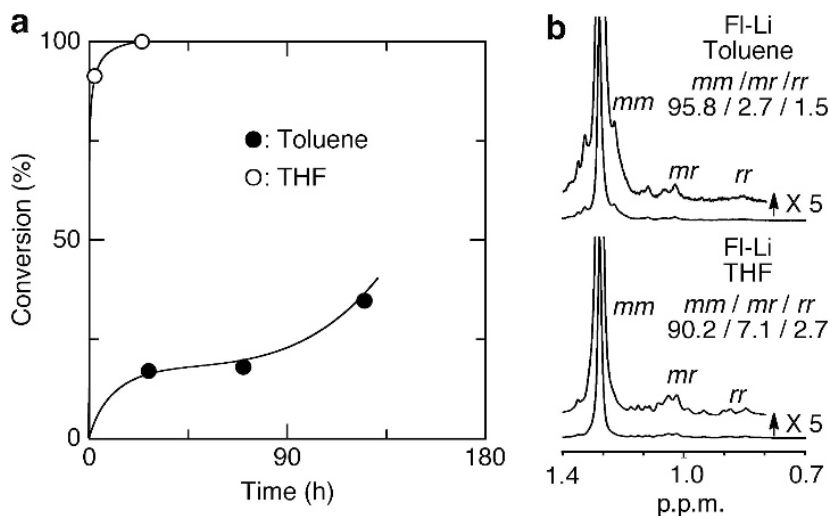

Figure 1 (a) Time-conversion curves for anionic polymerization of TTMSSMA in toluene or THF at $-78^{\circ} \mathrm{C}$ in the absence of chiral ligand and (b) ${ }^{1} \mathrm{H}$ NMR spectra of PMMA converted from the obtained poly(TTMSSMA): [TTMSSMA $]_{0}=0.50 \mathrm{~m}$; $[\mathrm{FI}-\mathrm{Li}]_{0}=10 \mathrm{~mm}$.

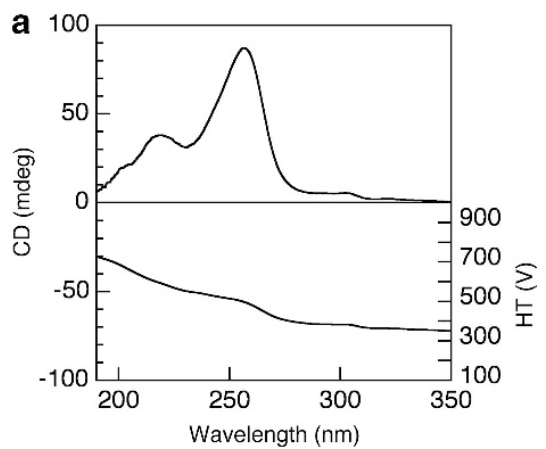

b

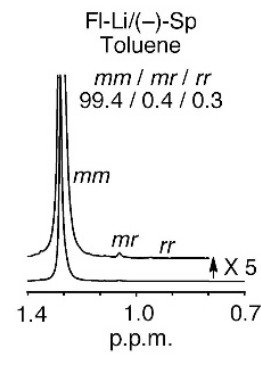

Figure 2 (a) DRCD spectrum of poly(TTMSSMA) obtained in the asymmetric anionic polymerization of TTMSSMA in toluene at $-78^{\circ} \mathrm{C}$ : $[\text { TTMSSMA }]_{0}=0.50 \mathrm{~m} ;[\mathrm{FI}-\mathrm{Li}]_{0}=10 \mathrm{~mm} ;[(-)-\mathrm{Sp}]_{0}=12 \mathrm{~mm}$. (b) ${ }^{1} \mathrm{H} \mathrm{NMR}$ spectrum of PMMA converted from the poly(TTMSSMA).

The poly(TTMMSMA) obtained with Fl-Li/( - )-Sp was characterized by DRCD, ${ }^{39,40}$ which can measure the chirality of solid-state samples (Figure 2a). The obtained poly(TTMMSMA) exhibited 

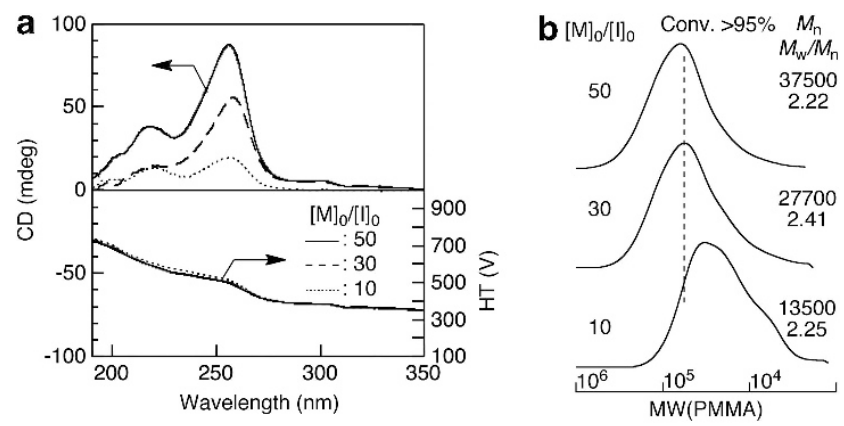

Figure 3 (a) DRCD spectra of poly(TTMSSMA) obtained in the asymmetric anionic polymerization of TTMSSMA at $-78^{\circ} \mathrm{C}$ : [TTMSSMA] $]_{0}=0.50 \mathrm{M}$; $[\mathrm{Fl}-\mathrm{Li}]_{0}=10-50 \mathrm{~mm}$; $[(-)-\mathrm{Sp}]_{0}=12-60 \mathrm{~mm}$. (b) Size-exclusion chromatography curves of PMMA converted from the poly(TTMSSMA).

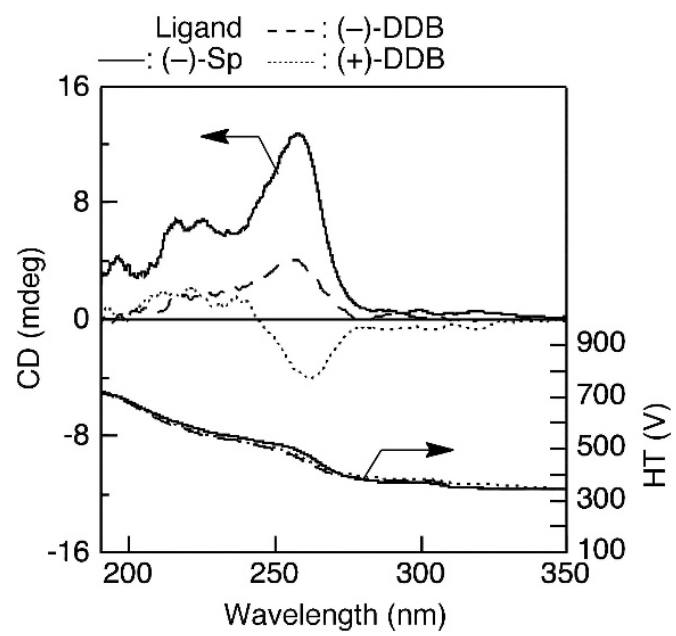

Figure 4 DRCD spectra of poly(TTMSSMA) obtained in the asymmetric anionic polymerization of TTMSSMA with $\mathrm{FI}-\mathrm{Li} /(-)-\mathrm{Sp},(-)-\mathrm{DDB}$, or (+)DDB in toluene at $-40{ }^{\circ} \mathrm{C}$ : $[\mathrm{TTMSSMA}]_{0}=0.50 \mathrm{M} ;[\mathrm{FI}-\mathrm{Li}]_{0}=10 \mathrm{~mm}$; [chiral ligand $]_{0}=12 \mathrm{~mm}$.

characteristic circular dichroism in the backbone region, that is, a carbonyl group signal at $220 \mathrm{~nm}$ and a supersilyl group signal at $255 \mathrm{~nm}$ originating from the $\sigma$-conjugation of the Si-Si groups. ${ }^{18}$ These chiralities arise from the helical main-chain conformation of the obtained poly(TTMMSMA), as they do for the other bulky poly(triarylmethyl methacrylates) obtained under similar conditions. ${ }^{13}$ The obtained polymer was further converted into PMAA and subsequently PMMA to analyze the tacticity and the molecular weights. Almost complete isotacticity $(m m=99 \%)$ was observed, as shown in Figure 2b.

The asymmetric polymerization was further investigated by varying the initial feed ratio of monomer to initiator ([TTMSSMA $]_{0} /[\mathrm{Fl}-$ $\mathrm{Li}]_{0}=50-10$, entries $4-6$ in Table 1). In all cases, the monomer was consumed quantitatively, yielding insoluble polymers. All of the obtained polymers showed similar patterns in the DRCD spectra, the intensity of which decreased by decreasing the feed ratio (Figure 3a). The molecular weights of PMMA converted from the obtained poly(TTMSSMA) decreased when the feed ratio of $[\text { TTMSSMA }]_{0} /[\mathrm{Fl}-\mathrm{Li}]_{0}$ decreased (Figure $3 \mathrm{~b}$ ), but the high isotacticity was maintained. These results indicate that $\mathrm{Fl}-\mathrm{Li}$ works as an initiator to induce the asymmetric anionic polymerization of TTMSSMA in the presence of (-)-Sp and yields the prevailing one-handed helical polymers. However, the initiation efficiencies were generally low, which can be attributed to the remaining lowmolecular weight oligomers that possess low reactivities because of the low tacticities, as reported in asymmetric anionic polymerization of TrMA. ${ }^{15}$

The effects of the polymerization temperature and the chiral ligand structure were also examined (entries 7-9 in Table 1). The use of $\mathrm{Fl}-\mathrm{Li} /(-)-\mathrm{Sp}$ at $-40^{\circ} \mathrm{C}$ also induced quantitative polymerization, yielding optically active and isotactic polymers with similar molecular weights. Although the polymer obtained at $-40^{\circ} \mathrm{C}$ exhibited a DRCD spectrum similar to that of the polymer obtained at $-78^{\circ} \mathrm{C}$, the intensity of the $-40^{\circ} \mathrm{C}$ polymer spectrum was lower (Figure 4). In addition, the isotacticity was slightly lower $(\mathrm{mm} / \mathrm{mr} /$ $r r=95.8 / 2.2 / 2.0)$. These results indicate that the isospecificity and the helix-sense selectivity decrease at higher temperatures. The Fl-Li/ $(+)-D D B$ and $(-)$-DDB systems led to quantitative polymerization at $-40{ }^{\circ} \mathrm{C}$, whereas no polymer was obtained with $\mathrm{Fl}-\mathrm{Li} /(+)-\mathrm{DDB}$ at $-78^{\circ} \mathrm{C}$, as described above. Both polymers obtained with the enantiomers at $-40^{\circ} \mathrm{C}$ under the same conditions showed similar molecular weights $\left(M_{\mathrm{n}}=6000-8000\right)$ and almost the same tacticities $(\mathrm{mm} / \mathrm{mr} / \mathrm{rr}=61 / 23 / 15)$, although the isotacticities were lower than that obtained with (-)-Sp at $-40^{\circ} \mathrm{C}$. The DRCD spectra of the two polymers were almost mirror images of each other, although their intensities were lower than that obtained with $(-)$-Sp. These results indicate again that TTMSSMA is polymerized by the chiral initiating systems to give the prevailing one-handed helical polymers, in which a helical conformation with excess right- or left-handed helicity is induced by the chirality of the ligands.

The chiral recognition ability of the optically active poly (TTMSSMA) was evaluated by the chiral adsorption method using Tröger's base and trans-stilbene oxide as the racemates for the polymers obtained with the $\mathrm{Fl}-\mathrm{Li} /(-)-\mathrm{Sp}$ system at $-78^{\circ} \mathrm{C}$. The optically active poly(TTMSSMA) adsorbed $52 \%$ and $19 \%$ of the Tröger's base and the trans-stilbene oxide, respectively. The e.e.'s in solution were very low $((+)-0.46 \%$ and $(-)-2.4 \%$, respectively) compared with that of optically active poly(TrMA), ${ }^{22}$ most likely because the almost nonpolar supersilyl group in the side chain of the helix cannot distinguish the chirality of these polar compounds.

\section{CONCLUSIONS}

The novel bulky silyl methacrylate TTMSSMA undergoes asymmetric anionic polymerization in the presence of appropriate chiral ligands, such as (-)-Sp, yielding optically active polymers with prevailing one-handed helical conformations, as observed for a series of bulky triarylmethyl methacrylates. This finding will contribute to further developments in asymmetric polymerization and in the development of helical polymeric materials based on the designed bulky monomers.

\section{ACKNOWLEDGEMENTS}

This work was supported in part by JSPS Research Fellowships for Young Scientists for KI (No. 22-8509), a Grant-in-Aid for Young Scientists (S) for MK (No. 19675003) by the Japan Society for the Promotion of Science, and the Global COE Program 'Elucidation and Design of Materials and Molecular Functions.' We thank Mr Muennori Shimoyama, Dr Hiroki lida and Professor Eiji Yashima (Nagoya University) for their technical support and useful suggestions on DRCD measurement. 
1 Okamoto, Y., Suzuki, K., Ohta, K., Hatada, K. \& Yuki, Y. Optically active poly(triphenylmethyl methacrylate) with one-handed helical conformation. J. Am. Chem. Soc 101, 4763-4765 (1979)

2 Okamoto, Y. \& Yashima, E. Asymmetric polymerization of methacrylates. Prog. Polym. Sci. 15, 263-298 (1990).

3 Okamoto, Y. \& Nakano, T. Asymmetric polymerization. Chem. Rev. 94, 349-372 (1994).

4 Okamoto, Y., Nakano, T., Habaue, S., Shiohara, K. \& Maeda, K. Synthesis and chira recognition of helical polymers. J. Macromol. Sci., Pure Appl. Chem. A34, 1771-1783 (1997)

5 Nakano, T. \& Okamoto, Y. Asymmetric polymerization of methacrylates. Macromol. Rapid Commun. 21, 603-612 (2000).

6 Nakano, T. \& Okamoto, Y. Synthetic helical polymers: conformation and function. Chem. Rev. 101, 4013-4038 (2001).

7 Okamoto, T. (-)-Sparteine: the compound that most significantly influenced my research. J. Polym. Sci.: Part A: Polym. Chem. 42, 4480-4491 (2004).

8 Wulff, G. Main-chain chirality and optical activity in polymers consisting of $\mathrm{C}-\mathrm{C}$ chains. Angew. Chem. Int. Ed. 28, 21-37 (1989).

9 Yuki, Y., Okamoto, Y. \& Okamoto, I. Resolution of racemic compounds by optically active poly(triphenylmethyl methacrylate). J. Am. Chem. Soc. 102, 6356-6358 (1980).

10 Okamoto, Y. \& Yashima, E. Polysaccharide derivatives for chromatographic separation of enantiomers. Angew. Chem. Int. Ed. 37, 1020-1043 (1998).

11 Yamamoto, S. \& Okamoto, Y. Optically active polymers for chiral separation. Bull. Chem. Soc. Jpn. 77, 227-257 (2004).

12 Ikai, T. \& Okamoto, Y. Structure control of polysaccharide derivatives for efficient separation of enantiomers by chromatography. Chem. Rev. 109, 6077-6101 (2009).

13 Okamoto, Y., Suzuki, K. \& Yuki, H. Asymmetric polymerization of triphenylmethy methacrylate by optically active anionic catalysts. J. Polym. Sci. Polym. Chem. Ed. 18, 3043-3051 (1980)

14 Okamoto, Y., Shohi, H. \& Yuki, H. Facile synthesis of (+)- and (-)-poly(triphenylmethyl methacrylate)s and their macromers. J. Polym. Sci., Polym. Lett. Ed. 21, 601-607 (1983)

15 Nakano, T., Okamoto, Y. \& Hatada, K. Asymmetric polymerization of triphenylmethy methacrylate leading to a one-handed helical polymer: mechanism of polymerization. J. Am. Chem. Soc. 114, 1318-1329 (1992).

16 Okamoto, Y., Nakano, T., Shikisai, Y. \& Mori, M. Stereospecific and asymmetric polymerization of 1-phenylbenzosuberyl methacrylate with radical and anionic initiators. Macromol. Symp. 89, 479-488 (1995).

17 Nakano, T., Okamoto, Y. \& Hatada, K. Asymmetric polymerization of triphenylmethy methacrylate using 9-alkyl-9-fluorenyllithium -( -)-sparteine complexes. influence of organolithium structure on the propagation stereochemistry. Polym. J. 27, 892-903 (1995).

18 Ren, C., Chen, F., Xi, F., Nakano, T. \& Okamoto, Y. Helix-sense-selective polymerization of phenyl[bis(2-pyridyl)]methyl methacrylate and chiral recognition ability of the polymer. J. Polym. Sci.: Part A: Polym. Chem. 31, 2721-2728 (1993).

19 Nakano, T., Matsuda, A., Mori, M. \& Okamoto, Y. Asymmetric anionic polymerization of 1-phenyldibenzosuberyl and 1-(2-pyridyl)dibenzosuberyl methacrylates and chira recognition ability of the obtained polymers. Polym. J. 28, 330-336 (1996).

20 Nakano, T., Taniguchi, K. \& Okamoto, Y. Asymmetric polymerization of diphenyl-3 pyridylmethyl methacrylate leading to optically active polymer with helical conformation and chiral recognition ability of the polymer. Polym. J. 29, 540-544 (1997)

$21 \mathrm{Wu}$, J., Nakano, T. \& Okamoto, Y. Asymmetric anionic polymerization of (2-flurophenyl)(4-fluoropheyl)(2-pyridyl)methyl methacrylate leading to a helical polymer. J. Polym. Sci.: Part A: Polym. Chem. 36, 2013-2019 (1998).
22 Nakano, T., Satoh, Y. \& Okamoto, Y. Asymmetric polymerization of 1-(3-pyridyl)dibenzosuberyl methacrylate and chiral recognition by the obtained optically active polyme having single-handed helical conformation. Polym. J. 30, 635-640 (1998).

23 Nakano, T., Ueda, K. \& Okamoto, Y. Helix-sense-selective polymerization of (1-methylpiperidin-4-yl)diphenylmethyl methacrylate by anionic and free radical catalyses. J. Polym. Sci.: Part A: Polym. Chem. 39, 1610-1614 (2001).

24 Nakano, T., Kinjo, N., Hidaka, Y. \& Okamoto, Y. Asymmetric anionic and free-radical polymerization of 10,10-dimethyl- and 10,10-dibutyl-9-phenyl-9,10-dihydroanthracen- 9-yl methacrylate leading to single-handed helical polymers. Polym. J. 33, 306-309 (2001).

25 Bock, H., Meuret, J. \& Rupper, K. 'Supersilyl' compounds $\left(\mathrm{R}_{3} \mathrm{Si}\right)_{3} \mathrm{SiSi}\left(\mathrm{SiR}_{3}\right)_{3}$ and $\left(\mathrm{R}_{3} \mathrm{Si}_{3} \mathrm{SiC}_{6} \mathrm{H}_{4} \mathrm{Si}\left(\mathrm{SiR}_{3}\right)_{3}\right.$ : structures and properties. Angew. Chem. Int. Ed. Engl. 32, 414-416 (1993).

26 Boxer, M. B., Albert, B. J. \& Yamamoto, H. The super silyl group in diastereoselective aldol and cascade reactions. Aldrichim. Acta 42, 1-15 (2009)

27 Boxer, M. B. \& Yamamoto, H. Remarkable tris(trimethylsilyl)silyl group for diastereoselective [2 + 2] cyclizations. Org. Lett. 7, 3127-3129 (2005).

28 Albert, B. J. \& Yamamoto, H. A triple-aldol cascade reaction for the rapid assembly of polyketides. Angew. Chem. Int. Ed. 49, 2747-2749 (2010).

29 Brady, P. B. \& Yamamoto, H. Rapid and stereochemically flexible synthesis of polypropionates: super-silyl-governed aldol cascades. Angew. Chem. Int. Ed. 51, 1942-1946 (2012)

30 Satoh, K. \& Kamigaito, M. Stereospecific living radical polymerization: dual control of chain length and tacticity for precision polymer synthesis. Chem. Rev. 109, 51205156 (2009).

31 Ishitake, K., Satoh, K., Kamigaito, M. \& Okamoto, Y. Stereospecific free radical and RAFT polymerization of bulky silyl methacrylates for tacticity and molecular weight controlled poly(methacrylic acid). Macromolecules 44, 9108-9117 (2011).

32 Tsuruta, T. \& Furukawa, J. Stereoregular polymerizations of acrylic esters and its related monomers. Bull. Inst. Chem. Res. Kyoto Univ. 40, 151-170 (1962).

33 Aylward, N. N. Stereoregular poly(methacrylic acids). J. Polym. Sci. A-1, 8 319-328 (1970).

34 Kamachi, M., Kurihara, M. \& Sitlle, J. K. Synthesis of block polymers for desalination membranes. preparation of block copolymers of 2-vinylpyridine and methacrylic acid or acrylic acid. Macromolecules 5, 161-167 (1972).

35 Kurihara, M., Kamachi, M. \& Sitlle, J. K. Synthesis of ionic block polymers for desalination membranes. J. Polym. Sci. Polym. Chem. Ed. 11, 587-610 (1973).

36 Morishima, Y., Hashimoto, T., Itoh, Y., Kamachi, M. \& Nozakura, S. Synthesis of amphiphilic block copolymers. block copolymer of methacrylic acid and 9-vinylphenanthrene. Macromol. Chem. Rapid Commun. 2, 507-510 (1981).

37 Kitayama, T., He, S., Hironaka, Y., lijima, T. \& Hatada, K. Preparation of highly stereoregular poly(methacrylic acid) by stereospecific anionic polymerization of trimethylsilyl methacrylate. Polym. J. 27, 314-318 (1995)

38 Kakehi, T., Yamashita, M. \& Yasuda, H. Synthesis of adhesion properties of nove syndiotactic block copolymers of alkyl (meth)acrylate with methacrylic acid and its analogues. React. Func. Polym. 46, 81-94 (2000)

39 Kuroda, R., Harada, T. \& Shindo, Y. A solid-state dedicated circular dichroism spectrophotometer: development and application. Rev. Sci. Instrum. 72, 3802 3810 (2001)

40 Harada, T., Miyoshi, Y. \& Kuroda, R. High performance diffuse reflectance circular dichroism spectrophotometer. Rev. Sci. Instrum. 80, 046101 (2009).

41 Baskaran, D. Strategic developments in living anionic polymerization of alky (meth)acrylates. Prog. Polym. Sci. 28, 521-581 (2003).

42 Okamoto, Y., Ohta, K., Hatada, K. \& Yuki, H. Anionic polymerization of triphenylmethy methacrylate. ACS Symp. Ser. 166, 353-365 (1981). 